CERNPPE/95-95

3 July 1995

\title{
HIGH RATE OPERATION OF MICRO-STRIP GAS CHAMBERS ON DIAMOND-COATED GLASS
}

\author{
R. Bouclier, M. Capeáns, G. Million, L. Ropelewski, F. Sauli and T. Temmel \\ CERN, Geneva, Switzerland \\ R. A. Cooke, S. Donnel and S.A. Sastri \\ SURMET Corp., Burlington MA, USA \\ N. Sonderer \\ IMT Masken und Teilungen AG, Greifensee, Switzerland
}

\begin{abstract}
Very high rate operation of micro-strip gas chambers can be achieved using slightly conducting substrates. We describe preliminary measurements realized with detectors manufactured on boro-silicate glass coated, before the photo-lithographic processing, with a diamond layer having a surface resistivity of around $10^{14} 1 / 2 / \square$. Stable medium-term operation, and a rate capability largely exceeding the one obtained with identical plates manufactured on uncoated glass are demonstrated. If these results are confirmed by long-term measurements, the diamond coating technology appears very attractive since it allows, with a moderate cost overhead, to use thin, commercially available glass with the required surface quality for the large-scale production of gas micro-strip detectors.
\end{abstract}

Submitted as letter to Nuclear Instruments and Methods in Physics Research 
With their high granularity and fast ions collection time, micro-strip gas chambers (MSGCs) are intrinsically capable of efficiently detecting and localizing very high rates of ionizing radiation. In early works it appeared however that use of a substrate with high resistivity, such as boro-silicate glass, results in a timedependent gain drop after applying the voltage caused by polarization of the dielectric. Moreover, already at moderate radiation fluxes, the proportional gain is reduced by a process of charge accumulation on the insulating surface between metal strips, with a considerable, rate-dependent decrease of performances [1]

The development of MSGCs manufactured on substrates with moderate resistivity allows to eliminate altogether polarization and surface charging-up effects; rate capabilities in excess of $10^{6} \mathrm{~mm}^{-2} \mathrm{~s}^{-1}$ have been demonstrated using electronconducting glass with resistivitv in the range $10^{9}$ to $10^{12} 1 / 2 \mathrm{~cm}$.

As an alternative to semi-conducting glass, rather difficult to procure in thin sheets, several groups have attempted to develop techniques of surface conditioning or coating to control the resistivity of insulating supports

6] In most cases, problems of residual reactivity of the thin layers with air or moisture, and damages resulting from the photo-lithographic manufacturing procedure have led to substantial modification of the electrical and morphological characteristics of the layers, and to instabilities of operation. For the easiest process to implement, over-coating of existing MSGC structures with a semi-conducting layer, severe long term damages under irradiation have been observed, possibly due to the interaction with ions and radicals in the avalanches [7]

We describe in the present work preliminary results of medium-term stability and high-rate behaviour of MSGCs manufactured on standard boro-silicate glass, coated before photo-lithography with a high resistivity, amorphous diamond-like carbon-based thin film. Several plates of $300 \mu \mathrm{m}$ thick boro-silicate glass varying in size from $100 \times 100 \mathrm{~mm}$ to $100 \times 250 \mathrm{~mm}^{2}$, commercially available with excellent surface quality ${ }^{1}$, have been coated on one side by with a thin $(\sim 1500 \AA)$ layer of diamond by low pressure plasma assisted chemical vapour deposition (LPCVD); the desired value of resistivity, around $10^{14} 1 / 2 / \square$, is obtained by doping, using a proprietary technology. Measurements on samples have shown a uniform resistivity within the measurement errors $(\sim 10 \%)$ over the surface of the plate; variations from sample to sample are of the same order. For checking both the value and the medium-term stability of the surface resistivity, we have evaporated coarse metal strip patterns, one $\mathrm{mm}$ apart, over the diamond-coated samples and monitored the current between strips as a function of time with a tera-ohmmeter ${ }^{2}$. Fig. 1 shows the remarkable stability in time of the surface resistivity, measured in air, over a period of three months; the difference of potential was kept constant and equal to $500 \mathrm{~V}$, the maximum allowed by the instrument. The average surface resistivity is $9 \cdot 10^{13} 1 / 2 / \square$, close to the target value. Essentially the same value $\left(1.2 \cdot 10^{14} 1 / 2 / \square\right)$ has been measured subsequently on the same sample kept for several days in dry nitrogen.

${ }^{1}$ DESAG D-263, made by Deutsche Spezialglas AG, Grünenplan, Germany

${ }^{2}$ Hewlett-Packard HP 4339A High Resisance Meter with HP 16339A Component Test Fixture 
We have tried various technologies for the manufacturing of MSGC plates on the diamond-coated support, a basic requirement being that no step in the photolithographic process removes or damages the layer. Due to several technological difficulties, the preferred candidates (with gold strips and manufactured by lift-off and galvanic growth) have not so far yielded plates of quality good enough to allow complete electrical testing. The most promising results have been obtained with plates manufactured by IMT with chromium by standard acid wet etching, a priori the most aggressive of the various technologies; we believe this to be due mostly to the well known superior quality of the chromium artwork. The note describes the preliminary results obtained with this technology; work is in progress to improve the quality of detectors manufactured with other photo-lithographic methods.

We have used one of our standard MSGC patterns (MS-6) having an active area of $80 \times 82 \mathrm{~mm}^{2}$; anode and cathode strips are $7 \mu \mathrm{m}$ and $100 \mu \mathrm{m}$ wide, respectively, at a distance centre to centre of $100 \mu \mathrm{m}$. The pattern is etched on a $4^{\prime \prime}$ by 4 " substrate coated with LPCVD diamond as described above. Groups of ten anodes are individually connected to the high voltage through a $500 \mathrm{k}^{1 / 2}$ protection resistor; signals from cathode strips are individually available, but for the present measurements we have ganged together groups of ten strips and connected the groups to charge amplifiers. The plates have no back-plane electrode, since this known to have very little or no influence on gain and rate capability when using resistive substrates Detectors are mounted in the "clean box" assembly, described elsewhere; the gas filling is argon-dimethvl ether (DME) in equal proportions

9]_. High voltage and signals are distributed by glass-metal feed-through. Because of the length of connections and the high capacitance of the group of strips, the signal-to noise ratio is not particularly good, but sufficient for gain studies when irradiating the detector with a collimated $6 \mathrm{keV}$ $X$-ray beam from a generator. We have used an amplifier chain with shaping time constants of around a $\mu$ s for pulse height analysis. Pile-up problems at high fluxes are reduced using a small area, typically $0.5 \mathrm{~mm}^{2}$, for the irradiation; at the highest fluxes a current measurement is used to extend the region covered by pulse height analysis.

At power on, and with 5 groups of 10 cathodes grounded, we measure on the plate a leakage current of $115 \mathrm{nA}$ at $580 \mathrm{~V}$, yielding an average resistivity of $\sim 1.8$ $10^{12} 1 / 2 / \square$, consistent with the values measured on samples before etching; both the leakage current and the gain show a small initial, rate independent increase, reaching about $10 \%$ after tens of minutes. We believe this to be due to uniform heating of the layer due to the leakage current (the power density is $\sim 10 \mu \mathrm{W} \mathrm{cm} \mathrm{cm}^{-2}$ ). This point, as well as the temperature dependence of the gain due both to gas density and surface resistivity changes, require more investigation.

Full curves in Fig. 2 show the absolute gain and the counting rate plateau, measured at low flux, and the noise rate at fixed threshold. The gain is estimated from the pulse height measurement using the normalization procedure described in Ref.

$$
[9]
$$

Comparison with the measurements realized with an identical structure on standard D-263 glass (dashed curves in Fig. 2) shows that a slightly higher voltage (about 50 $\mathrm{V}$ ) is required to obtain the same gain with the coated chamber; this is the expected 
effect of the field modification due to surface currents
$[10]$
. Although we have not attempted to reach the highest gains at this point, the similar shapes of the noise curves suggests that the maximum gain one can reach before discharge in the diamond-coated detector is the same as in the uncoated plate [11]

Gradually increasing the X-ray flux, we have recorded both current and pulse height on the detector. Fig. 3 shows the relative gain measured as a function of rate, at increasing values of flux; the avalanche size for the measurement is about $4 \cdot 10^{5}$ electrons (an absolute gain of 2000), and the drift field $3.5 \mathrm{kV} \mathrm{cm}-1$. There is no indication of rate-dependent gain drop up to the highest rate that could safely be recorded in the pulse height mode. For comparison, we have reproduced in the figure the measurements realized in identical conditions on a standard D-263 plate without back-plane; the gain drops by about 30\% already at the first increase of rate $^{3}$.

In Fig. 4, the normalized gain is plotted as a function of rate in a wider range of X-rays flux. Data points below $10^{5} \mathrm{~mm}^{-2} \mathrm{~s}^{-1}$ are deduced from pulse height measurement, while for the points above we have used the measurement of current (after subtraction of the leakage). For comparison, the behaviour of a standard, uncoated D-263 plate without back-plane is also shown. The detector manufactured on the coated glass exhibits a stable operation up to rates in excess of $10^{6} \mathrm{~mm}^{-2} \mathrm{~s}^{-1}$.

No change in gain and performances has been observed with the detector continuously irradiated for one week to an X-ray flux of $10^{4} \mathrm{~mm}^{-2} \mathrm{~s}^{-1}$, at an avalanche size of $2 \cdot 10^{5}$. Longer term irradiations are on the way.

We conclude that high-resistivity LPCVD diamond coating seems a valuable solution to largely improve the stability of operation and the rate capability of

3 A higher rate capability can be obtained at higher drift fields and with a back-plane electrode kept at appropriate potential; this however increases the cost of manufacturing, and results in a decrease of the maximum achievable gain, as discussed for example in Ref.

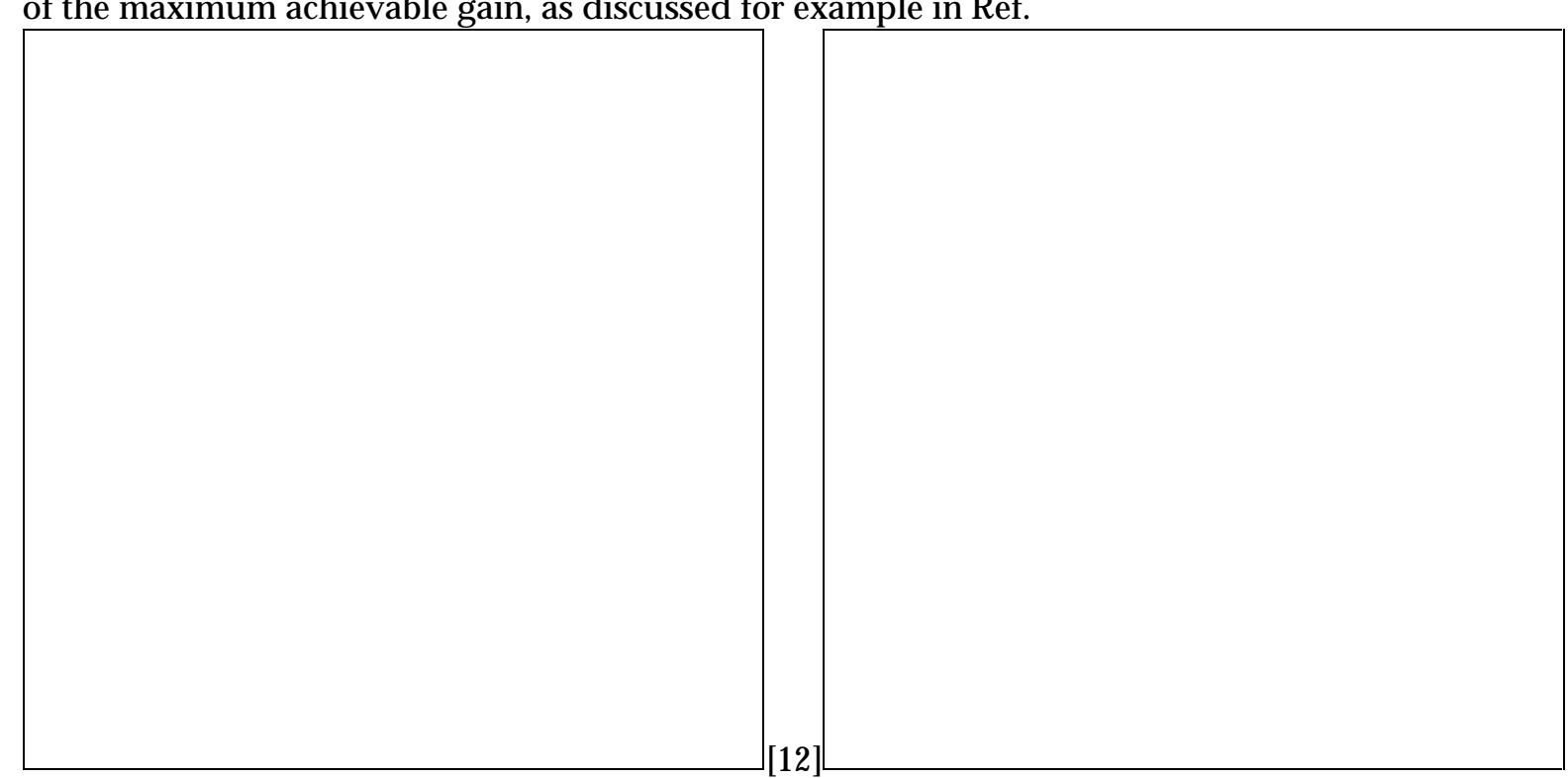


MSGC detectors. Standard chromium wet etching photo-lithography can been used without noticeable modification of the surface resistivity; general operating characteristics of the plates (gain and noise) are preserved and satisfy the stringent demands of advanced detectors. Due to its low intrinsic conductivity, chromium can be used only up to strips' length of perhaps $10 \mathrm{~cm}$, with a signal attenuation of 20$30 \%$ that can probably be tolerated in tracking devices

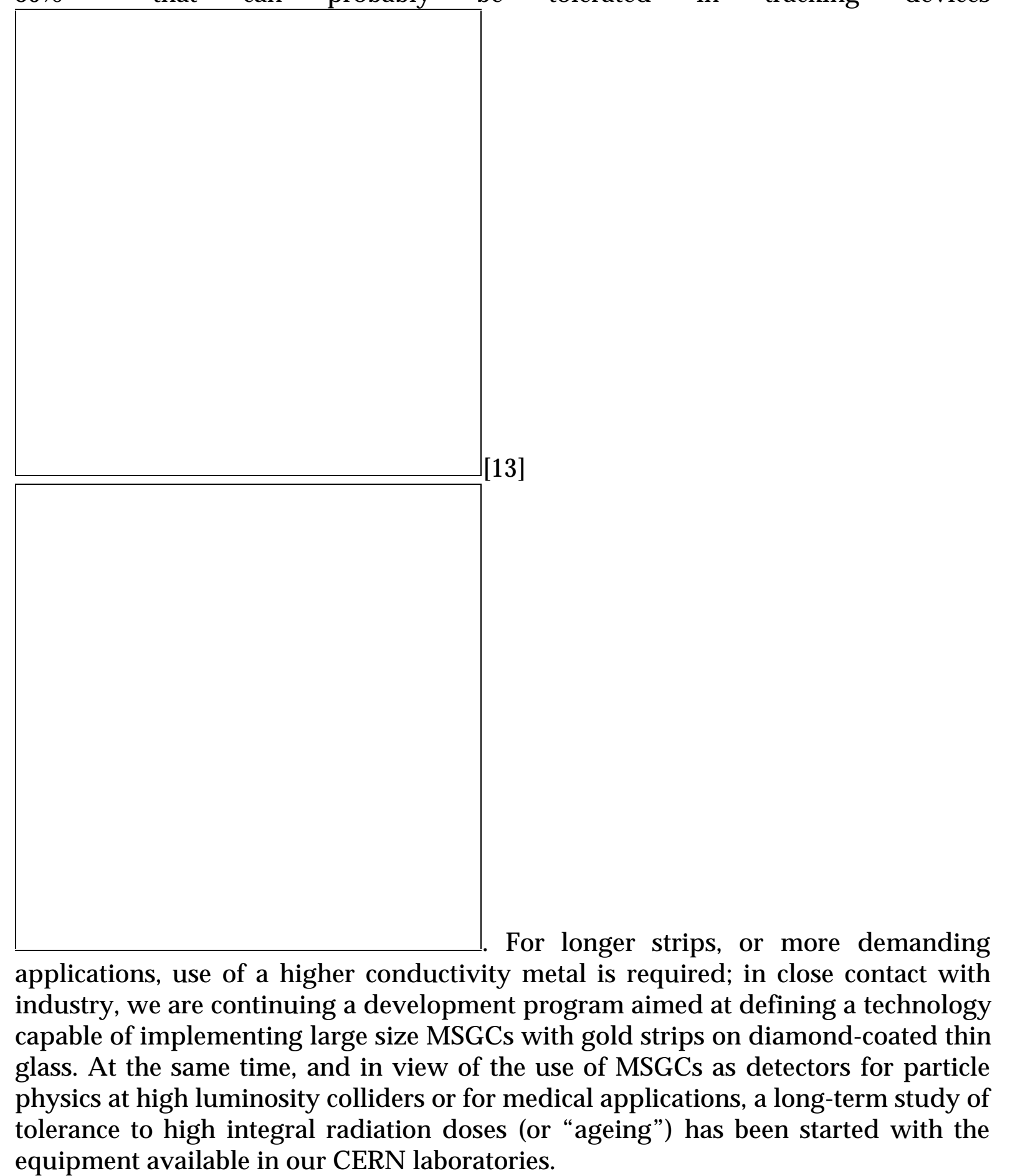

The use of resistive diamond coating to solve charging-up problems in MSGCs was suggested to one of us (FS) by David Anderson. His help in establishing the industrial contacts leading to the present work is here acknowledged. 


\section{REFERENCES}

[1] R. Bouclier, J.J. Florent, J. Gaudaen, G. Million, A. Pasta, L. Ropelewski, F. Sauli, and L.I. Shekhtman, Nucl. Instrum. Methods A323 (1992) 240.

[2] R. Bouclier, G. Million, L. Ropelewski, F. Sauli, Y. Pestov, and L.I. Shekhtman, Nucl. Instrum. Methods A332 (1993) 100.

[3] A. Pallares, S. Barthe, A.M. Bergdolt, R. Blaes, J.M. Brom, J. Cailleret, E. Christophel, J. Coffin, H. Eberle, R. Fang, J.C. Fontaine, W. Geist, T. Kachelhoffer, H. Kettunen, V. Mack, A. Michalon, A. Romero, J.L. Riester, J.P. Schunck, M.H. Sigward, et al., CRN 93-64 (1993).

[4] S. Brons, W. Bruckner, M. Heidrich, I. Konorov, and S. Paul, Nucl. Instrum. Methods A342 (1994) 411.

[5] M. Salomon, J. Armitage, M. Dixit, W. Faszer, D. Lam, and G. Oakham, IEEE Trans. Nucl. Sci. NS-41 (1994) 817.

[6] R. Bouclier, C. Garabatos, G. Manzin, F. Sauli, L. Shekhtman, T. Temmel, G.Della Mea, G.Maggioni, V.Rigato, and I.Logachenko, IEEE Trans. Nucl. Sci. NS-41 (1994) 821.

[7] R. Bouclier, M. Capeáns, C. Garabatos, G. Manzin, G. Million, L. Ropelewski, F. Sauli, E. Shefer, L. Shekhtman, T. Temmel, G.D. Mea, G. Maggioni, and V. Rigato, Proc. Int. Workshop. on Micro-Strip Gas Chambers, Legnaro, 1994 (Ed. Progetto, Padova 1995) 173.

[8] T. Beckers, R. Bouclier, C. Garabatos, G. Million, F. Sauli, and L. Shekhtman, Nucl. Instrum. Methods A346 (1994) 95.

[9] R. Bouclier, C. Garabatos, G. Manzin, G. Million, F. Sauli, T. Temmel, and L. Shekhtman, Nucl. Instrum. Methods A348 (1994) 109.

[10] J.J. Florent, J. Gaudaen, L. Ropelewski, and F. Sauli, Nucl. Instrum. Methods A329 (1993) 125.

[11] R. Bouclier, M. Capeáns, C. Garabatos, G. Manzin, G. Million, L. Ropelewski, F. Sauli, T. Temmel, L. Shekhtman, V. Nagaslaev, Y. Pestov, and A. Kuleshov, CERN-PPE/95-36 (1995). Subm. Nucl. Instrum. Methods

[12] J. Bohm, R. Bouclier, M. Capeans, C. Garabatos, G. Manzin, G. Million, F. Sauli, T. Temmel, and L. Shekhtman, Nucl. Instrum. Methods A360 (1994) 34. 
[13] R. Bouclier, M. Capeáns, C. Garabatos, G. Manzin, A. Peisert, L. Ropelewski, F. Sauli, J.C.Santiard, L.I.Shekhtman, T.Temmel, and G. Fischer, Proc. Int. Workshop. on Micro-Strip Gas Chambers Legnaro, 1994 (Ed. Progetto, Padova 1995)

79.

\section{FIGURE CAPTIONS}

Fig. 1: Stability in time of the surface resistivity of a 4 " by 4 " glass plate coated with high-resistivity diamond, measured in air at a field of $500 \mathrm{~V} \mathrm{~mm}^{-1}$.

Fig. 2: Gain, counting rate and noise rate measured at low source intensity on MSGC plates manufactured in chromium on standard D-263 glass (dashed curves) and diamond-coated D-263 (full curves). Gas filling argon-DME (50-50).

Fig. 3: Relative gain of MSGCs manufactured on standard and diamond-coated glass, measured stepping up the X-ray flux. Avalanche size: $4 \cdot 10^{5}$, gas filling A-DME (50-50).

Fig. 4: Rate dependence of the relative gain measured on standard and diamondcoated plates. Above $10^{5} \mathrm{~Hz} \mathrm{~mm}$, the gain is deduced from a current measurement; below, from pulse height. The drift field is $3.5 \mathrm{kV} \mathrm{cm}-1$. Both plates have no backplane electrode. 


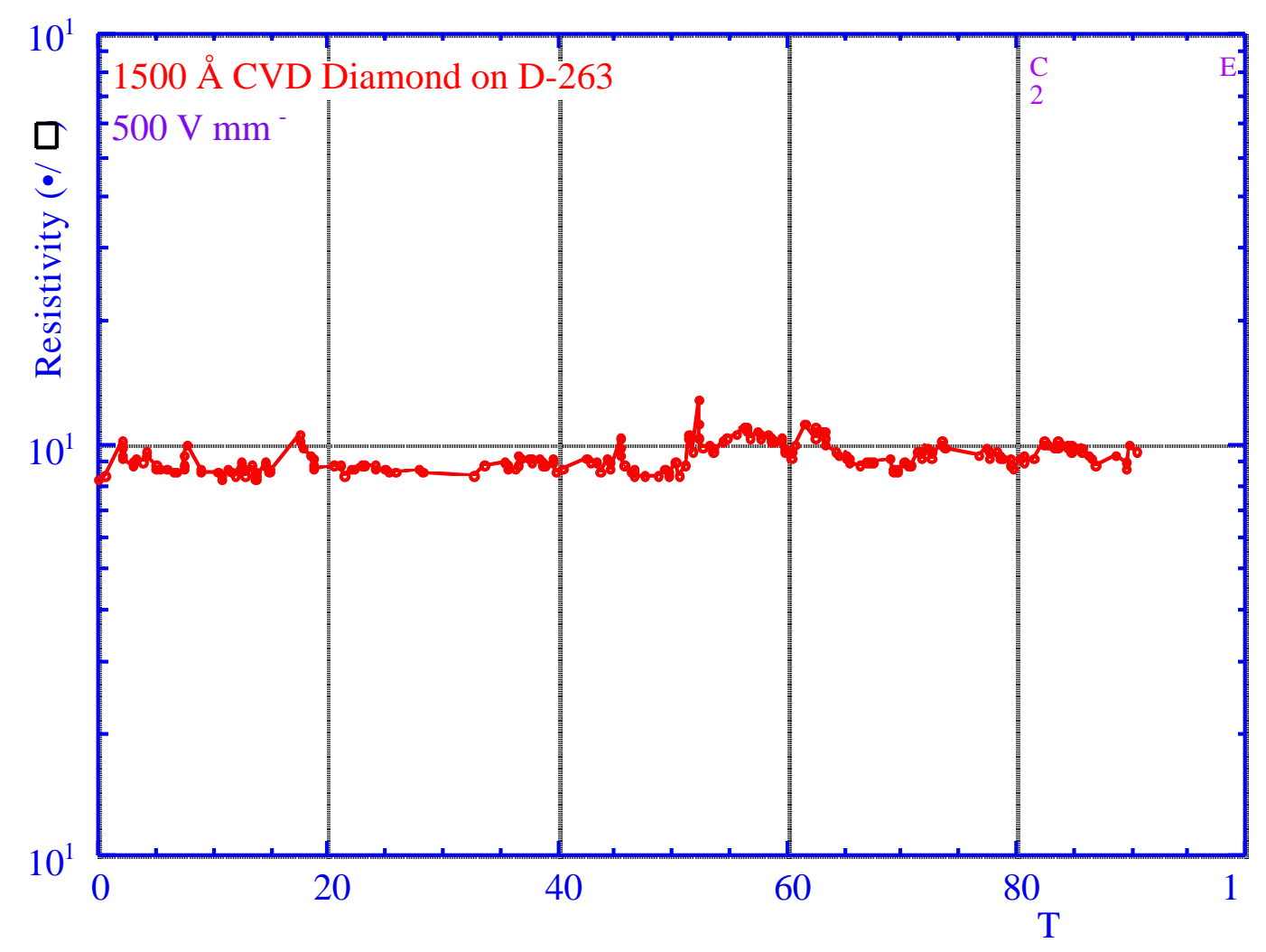

Fig. 1

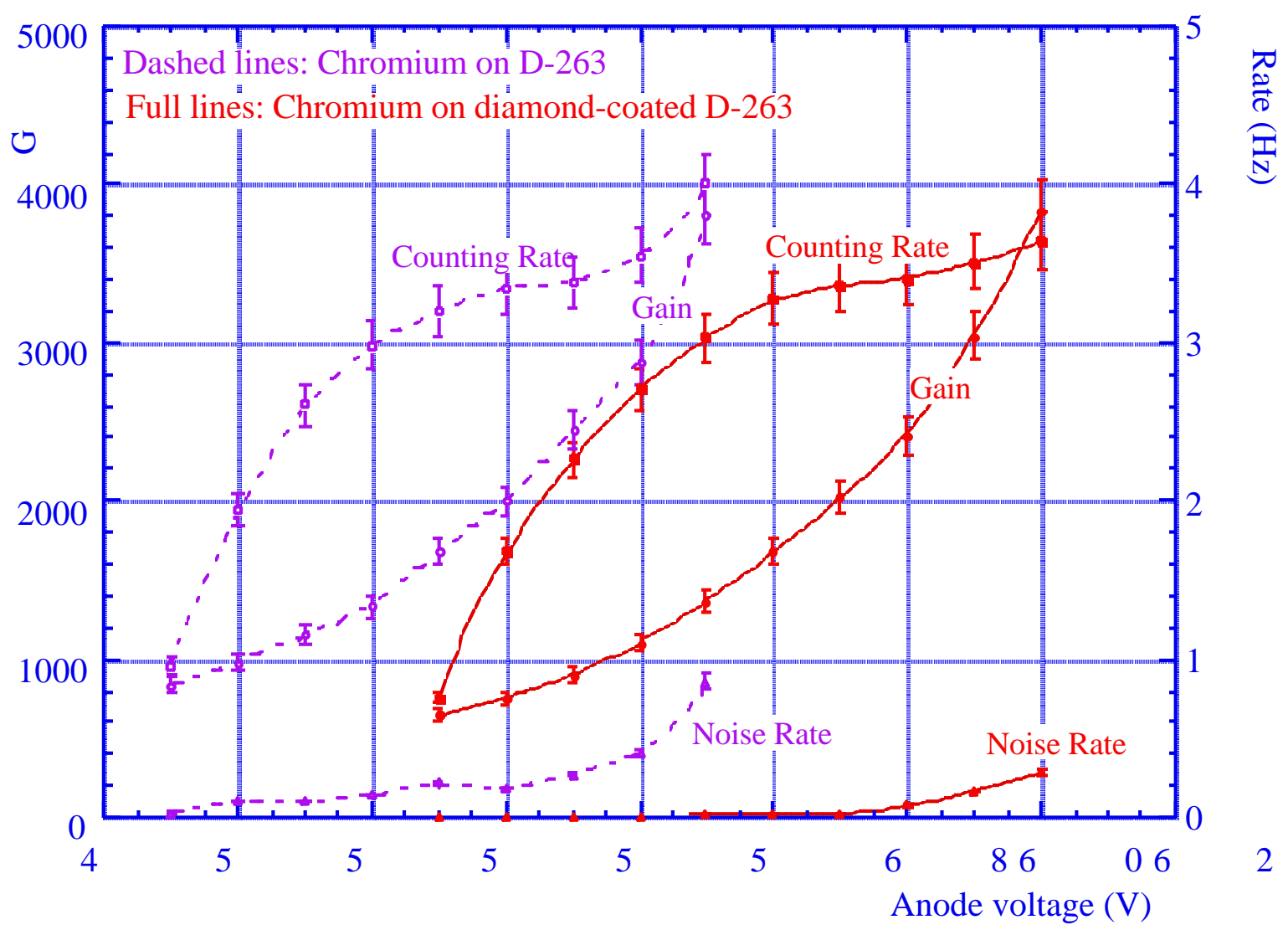

Fig. 2 


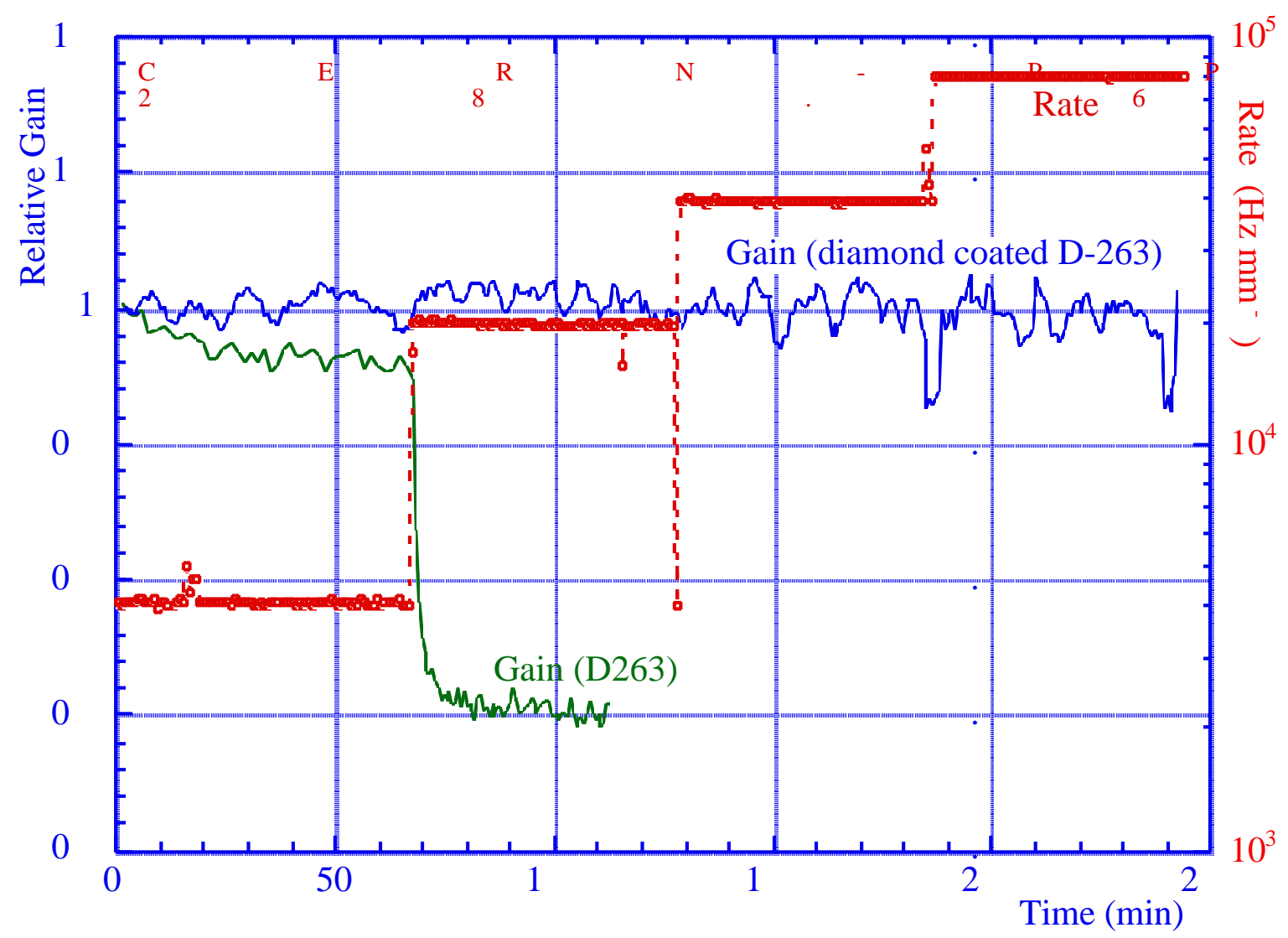

Fig. 3

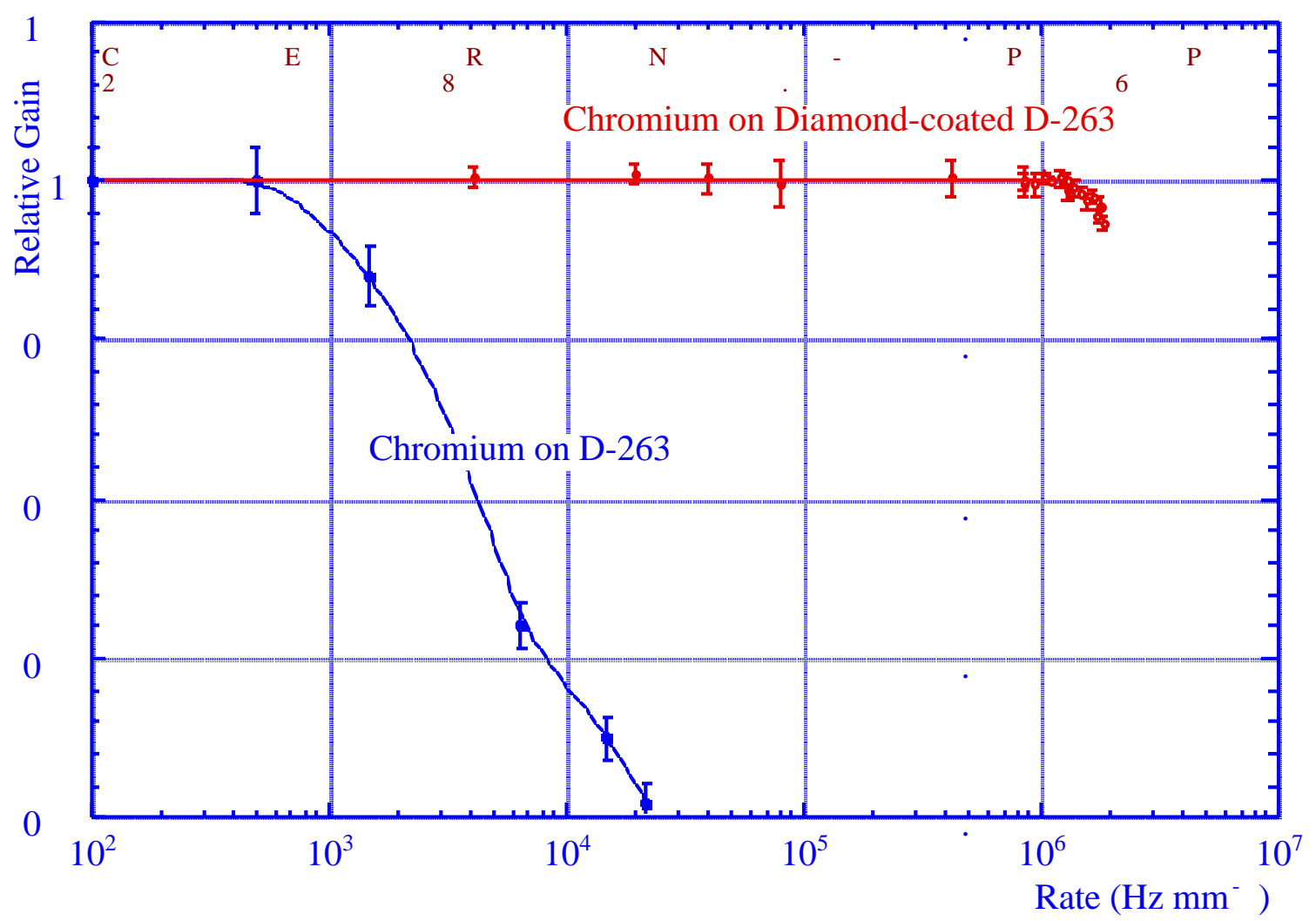

Fig. 4 